\title{
MDCT Diagnosis of Isolated Colonic Hernia Through the Esophageal Hiatus
}

\author{
Naime Altınkaya, Zafer Koç, Özlem Alkan, Şenay Demir
}

Department of Radiology, Adana Teaching and Research Hospital, Başkent University, Adana, Turkey

\begin{abstract}
Colonic herniation through the esophageal hiatus is an unusual condition. Isolated trans-hiatal herniation of the transverse colon is very rare and only five cases have been reported. All these reported patients were symptomatic, and had dysphagia, epigastralgia, vomiting, dyspnea, cough, or palpitations. Hiatal hernia is an important clinical problem. Because of the severe complications of hiatal hernia, including bleeding, strangulation, and perforation, early diagnosis and treatment are important. A 75-year-old man who had prostate carcinoma was evaluated with multidetector computed tomography (MDCT). In this case, CT findings and clinical emphasis of the incidentally discovered isolated transverse colonic hiatal herniation was reviewed.
\end{abstract}

Key Words: Esophageal hiatus, hernia, multidetector computed tomography (MDCT)

Received: 06.02.2009

Accepted: 07.03.2009

\section{Introduction}

Esophageal hiatal hernias are classified into four types anatomically. A type IV hiatal hernia is associated with migration of the stomach and other organs, such as the colon and small intestine. However, a solitary organ other than the stomach rarely migrates through the hiatus (1). Isolated trans-hiatal herniation of the colon is very rare; only five cases have been reported (1-4). All these reported patients were symptomatic. Our patient was asymptomatic and his hernia was discovered incidentally on abdominal computed tomography (CT) examination.

\section{Case Report}

A 75-year-old man was admitted to our hospital for a prostate carcinoma evaluation. He was referred to our radiology unit for CT evaluation. The patient did not have any symptoms of esophageal or colonic obstruction, dysphagia, or reflux. The patient had no history of trauma.

Portal phase images were obtained using a four-channel multidetector computed tomography (MDCT) scanner. The scanning parameters were contiguous $2.5 \mathrm{~mm}$ collimation with a pitch value of 1.25 , with 5 -mm-thick slices reconstructed secondarily at $1-\mathrm{mm}$ intervals. The images were analyzed on a workstation using post-processing (three-dimensional reconstructions). CT of the abdomen showed herniation of the mid-segment of the transverse colon into the mediastinum via a wide esophageal hiatus. The esophagogastric junction and stomach were in their normal locations (Figs. 1 and 2). PA chest $x$-ray showed bilateral paracardiac radiopacity consistent with hiatal hernial sac (Figur 3). The patient underwent open transabdominal surgery. The hernial sac was dissected from the mediastinal attachments and replaced in the abdomen. A Nissen fundoplication was performed.

\section{Discussion}

Esophageal hiatal hernias are classified into four types anatomically. In a sliding or type I hiatal hernia, the esophagogastric junction slides into the mediastinum above the diaphragm. In a paraesophageal or type II hernia, the esophagogastric junction is in its normal location, but the gastric fundus 'rolls' into the mediastinum. A paraesophageal hernia with a sliding component is often called a type III (mixed) hernia. In a type IV hiatal hernia, the esophagogastric junction is located normally, but the stomach has migrated along with other organs, such as the colon and small intestine. However, a solitary organ other than the stomach rarely migrates through the hiatus (1).

Esophageal hiatal hernias are commonly associated with the intrathoracic migration of the stomach (1). CT reveals extension of a portion of the proximal stomach into the lower middle mediastinum. This acquired abnormality is secondary to laxity and stretching of the phrenoesophageal ligament and widening of the esophageal hiatus. Obesity and increased intra-abdominal pressure are contributing factors. Patients may be asymptomatic or have symptoms of reflux. Very large hernias can become incarcerated or undergo volvulus (5).

This case was presented as a poster at the $27^{\text {th }}$ Turkish National Radiology Congress, October 11-15, Antalya, 2006.

Address for Correspondence: Dr. Naime Altınkaya, Department of Radiology, Adana Teaching and Research Hospital, Başkent University, Adana, Turkey Phone: +90322327 2727 E-mail: naimeto@yahoo.com 


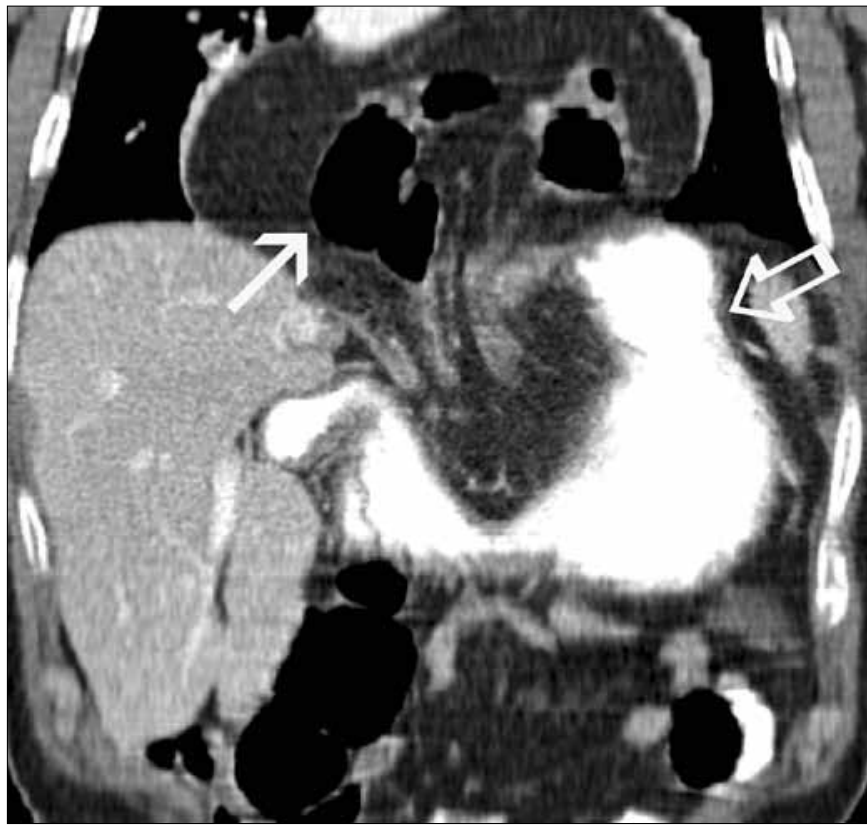

Figure 1. CT coronal curved planar reconstruction showing isolated herniation of the mid-part of the transverse colon into the mediastinum via a wide esophageal hiatus (arrow). The esophagogastric junction and stomach are located normally (open arrow)

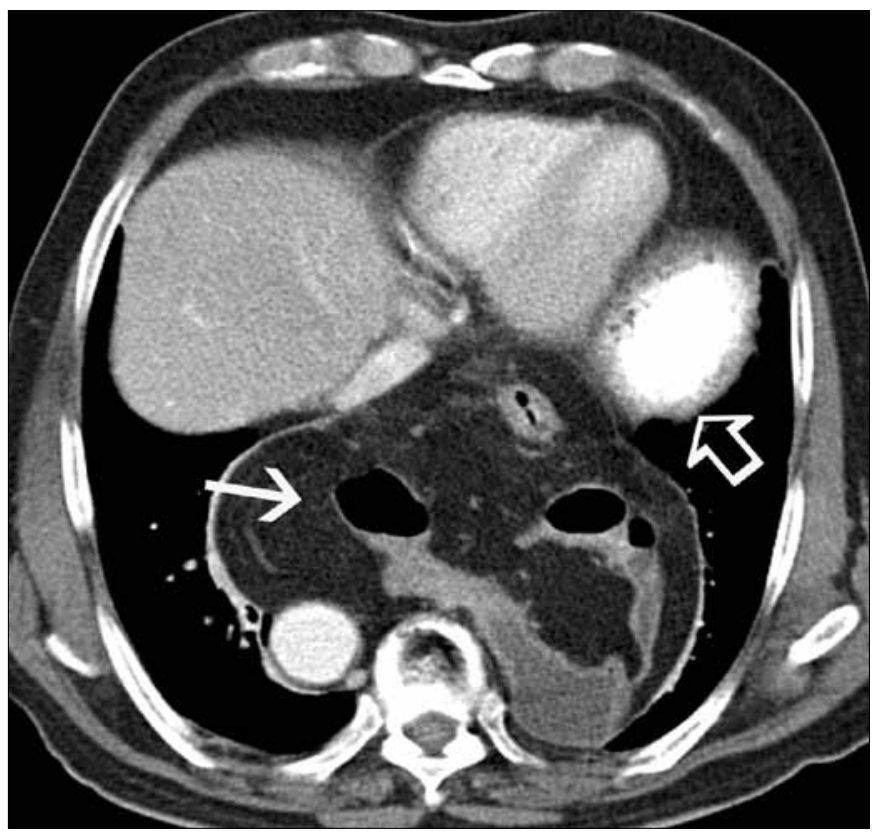

Figure 2. Axial CT image showing the transverse colon segment in the mediastinal hernia sac (arrow). The gastric fundus is seen in its normal location (open arrow)

With a paraesophageal type hiatal hernia, the stomach and other organs (small or large intestine) migrate into the mediastinum. However, trans-hiatal herniation of the transverse colon in the absence of an intrathoracic stomach is very rare. There have been only five reported cases. All of these reported patients were symptomatic, and had dysphagia, epigastralgia, vomiting, dyspnea cough, or palpitations $(1,4)$. Our patient

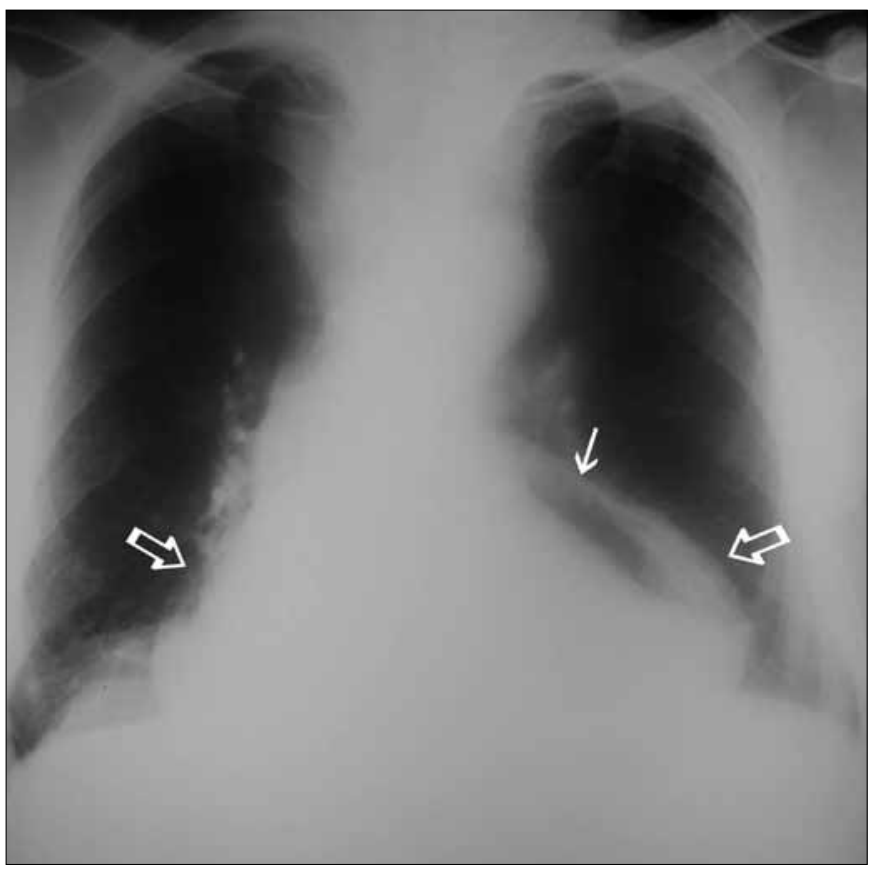

Figure 3. PA chest $x$-ray showed bilateral paracardiac radiopacity consistent with hiatal hernial sac (open arrows). A radiolucent area consistent with colonic gas (arrow). was also seen

was asymptomatic and his hernia was discovered incidentally on abdominal CT.

To our knowledge, this is the first reported case of an isolated colonic hiatal herniation diagnosed incidentally using MDCT. MDCT with multiplanar reformation can provide an excellent view of anatomic structures and allows a definitive diagnosis of esophageal hiatal herniation. An abnormally wide esophageal hiatus may be seen if it is oriented appropriately in the transverse plane.

In conclusion, an isolated colonic hernia through the hiatus may be asymptomatic, and MDCT with coronal reformatted images is a very effective, useful imaging technique for making the diagnosis.

\section{Conflict of Interest}

No conflict of interest was declared by the authors.

\section{References}

1. Tabira Y, Yoshida Y, Sakaguchi T, Yoshimatsu S. Isolated colonic hernia through the esophageal hiatus. Diseases of the Esophagus 2005;18:283-6.

2. Curtis D J, Brenza S J. Isolated herniation of the colon through the esophageal hiatus: case report. Mil Med 1984;149:515-8.

3. Felsher J A, Brodsky J, Brody F. Isolated trans-hiatal colonic herniation. J Laparoendosc Adv Surg Tech 2003;13:105-8.

4. Itabashi Y, Baba T, Kato S. A case of esophageal hiatal hernia with incarcerated transverse colon. Jpn J Gastroenterol Surg 2004; 37:479-82

5. Slone RM, Gierada DS Pleura, chest wall, and diaphragm. In: Lee JKT, Sagel SS, Stanley RJ and Heiken JP, editors. Computed Body Tomography with MRI Correlation. 3rd ed. New York: LippincottRaven; 1998. p. 455-520. 